\title{
Awake office based trans nasal flexible endoscopic diode laser assisted posterior cordotomy for bilateral vocal fold paralysis
}

\begin{abstract}
Purpose: The purpose of this retrospective study is to describe our experiencewith the new technique of Awake office based flexible endoscopic diode laser assisted posterior cordotomy for bilateral vocal fold paralysis. The technique was primarily designed as an alternative to traditional micro suspension $\mathrm{CO}_{2}$ posterior cordotomy for patients of bilateral vocal fold paralysis when associated with co morbidity leading to either difficulty for suspension laryngoscopy or high risk for general anesthesia.
\end{abstract}

Material and methods: The study was carried out on 20 patients of Bilateral Vocal Fold Paralysis associated with co morbidity with limitations for Micro suspension cordotomy. 12 patients were females and 8 patients were males in the age group of 25 and 75 years, attending Sri Sathya Sai E.N.T Hospital and Research Center for Voice Disorders, India from January 2012 to January 2017. All the patients were operated by posterior cordotomy done in office based setup under local anesthesia using Fiberoptic fiberoptic flexible laryngoscope and diode fiberlaser.

Results: Results proved the efficacy of the procedure based on the pre and postoperative Pulmonary Function Tests and Voice Handicap Index scores which were statistically significant.

Conclusion: The new technique was proved as safe and perfect alternative to traditional micro suspension cordotomy in patients of bilateral vocal fold paralysis associated with comorbities leading to either difficulty for suspension laryngoscopy or high risk for general anesthesia. The study also described it's advantages such as conservation of voice by avoiding the over correction of glottic space by monitoring the voice intraoperatively and feasibility for revision cordotomy in the Office basis.

Keywords: bilateral vocal fold paralysis, airway, diode laser, office based laryngeal surgery, voice, stridor, posterior cordotomy, awake surgeries
Volume 12 Issue 4 - 2020

\author{
Phaniendrakumar. V, Ramachandrareddy. S, \\ Ravindranath. T.A, Krishnamohan. K \\ Department of Laryngology, Sri Sathya Sai E.N.T Hospital, India
}

Correspondence: Phaniendrakumar. V, Director and Chief Laryngologist, Sri Sathya Sai E.N.T Hospital\& Research Center for Voice Disorders, 4/2 Brodiepet, Guntur 522002,A.P, India, Email drvoicep@hotmail.com

Received: August 19,2020 | Published: August 31, 2020
Abbreviations: BVFP, bilateral vocal fold paralysis; DLE, difficult laryngeal exposure; HRGA, high risk for general anaesthesia; PIF, peak inspiratory flow rate; VHI, voice handicap index

\section{Introduction}

The most common cause of Bilateral vocal fold paralysis (BVFP) is iatrogenic or surgical trauma commonly occurring after thyroidectomy (44\%), and other causes include malignancies (17\%), endotracheal intubation $(15 \%)$, neurologic causes $(12 \%)$, and idiopathic cases $(12 \%) .{ }^{1}$ Even today the treatment of bilateral vocal cord paralysis continues to be a challenging problem to the otolaryngologists. Almost all the patients with bilateral abductor paralysis have stridor. This may manifest immediately especially after thyroidectomy or may be insidious and precipitated later by an upper respiratory tract infection. For several years tracheotomy remained as only option for maintaining satisfactory airway. The management of BVFP continues to be challenging problem searching for a compromise between socially satisfactory phonation and an airway compatible with an active life. ${ }^{2}$ Several surgical procedures, have been proposed to improve the patient's airway insufficiency with acceptable voice. In 1922, Chevalier Jackson performed the first surgical procedure ventriculocordectomy ${ }^{3}$ for bilateral vocal fold immobility. In 1939, King suggested extra laryngeal arytenoidectomy. ${ }^{4}$ Suture lateralization of vocal fold ${ }^{5}$ was described in 1984, which was recommended mostly for revision cases in which additional lateralization of the true vocal fold was desired. Endoscopic arytenoidectomy ${ }^{6}$ was developed by Thornell WC in 1948. Ossoff et al in 1983 have mentioned the use of $\mathrm{CO}_{2}$ laser in endoscopic arytenoidectomy. ${ }^{7}$ In 1989, Dennis and Kashima introduced endoscopic carbon dioxide laser posterior cordotomy. ${ }^{8}$ Other techniques like PCA muscle reinnervation, electrical stimulation of the laryngeal muscles, and use of the Cummings mechanical device are experimental but promising. All surgical procedures lead to breathy voice except re-innervation procedures. The decision as to which of these several procedures should be undertaken is determined by the patient's individual requirements and the surgeon's preference. On the basis of the surgeon's clinical judgment, tracheotomy for patients with deteriorating airway should be initiated immediately. When treating patients of BVFP, if the patient does not require a tracheotomy for a significantly compromised airway, the first procedure can be unilateral posterior cordotomy. ${ }^{9}$ Various methods of endoscopic cordotomy has been described by different surgeons using Diathermy, ${ }^{10}$ Radiofrequency ${ }^{11}$ and Coblation. ${ }^{12}$ Ultimately unilateral $\mathrm{CO}_{2}$ laser posterior cordotomy under general anaesthesia 
as been proved as an effective and satisfactory procedure in treatment of bilateral vocal fold abductor paralysis because of its precise cut, safety, short operative time, satisfactory results, and low complication incidence. ${ }^{13}$ It produces satisfactory glottic space without aspiration and with only mild to moderate dysphonia .Microsuspension $\mathrm{CO}_{2}$ laser posterior cordotomy under general anaesthesia has been popularized as gold standard in the surgical management of Bilateral Vocal Fold Paralysis (BVFP). But these procedures have limitations when BVFP associated with co-morbid conditions leading to either Difficult Laryngeal Exposure (DLE) or High Risk for General Anaesthesia (HRGA) Carbon dioxide laser has been predominantly used since the introduction of laser surgery. However, there are some disadvantages of the $\mathrm{CO}_{2}$ device, namely, its straight-line beam delivery from surgical microscope, narrow penetration depth to tissue, difficult transportation, and increased cost. In addition, it has short penetration in tissues (0.1-0.3 $\mathrm{mm}$ on average), which reduces effective sealing of vessels (it coagulates vessels of calibers smaller than $0.5 \mathrm{~mm}$ ), thus, increasing bleeding during surgery. ${ }^{14}$ Most institutions continued using the $\mathrm{CO}_{2}$ laser, due to doctors' familiarity with its use, capabilities, and limitations. ${ }^{15}$ Next to $\mathrm{CO}_{2}, 980 \mathrm{~nm}$ diode laser is a new technology commonly applied for posterior cordotomy. Some authors, confer that diode laser has surgical precision only slightly inferior to the $\mathrm{CO}_{2}$ laser, but with a superior coagulation capability. Use of diode laser by contact makes it much safer than other laser sources by avoiding damage due to "beam escape" in an open field. ${ }^{16}$ For Diode Laser the preferred wavelength is $980 \mathrm{~nm}$ and fiber diameter is $300 \mu \mathrm{m}$ with output power varying from $3 \mathrm{~W}$ to $6 \mathrm{~W}$.

The endoscopic posterior cordotomy performed by contact diode laser is an effective and reliable method for the treatment of dyspnoea secondary to bilateral laryngeal paralysis, guaranteeing a sufficient airway without impairing swallowing and maintaining acceptable voice quality. ${ }^{17}$ Other advantages of laser posterior cordotomy with diode laser are short operative time and relative ease of the procedure, minimal bleeding and edema. ${ }^{18}$ Certain difficulties or limitations for micro laryngeal suspension endoscopic cordotomy have been described particularly when general anesthesia is a threat to the patient's life as in cases of recent infarct, aneurysms, severe bradycardia and seriously compromised cardiopulmonary conditions. ${ }^{19}$ The technique was also found difficult to apply in patients in whom it was impossible to extend the neck and difficult to identify the vocal cords, as in patients with short and thick neck, fracture \& fixation of cervical spine, ankylosing spondylitis mandibular (Prognathism), T.M.Joint deformities and contracted mouth etc. ${ }^{19,20}$ Until now external procedure under local anesthesia is the only choice left in such patients. Main disadvantages of external procedure are poor voice outcome and the need for neck incision. ${ }^{21}$ In such situations the present innovative technique of Awake office based flexible endoscopic diode laser assisted posterior cordotomy under local anesthesia can be a suitable alternative to conventional micro suspension endoscopic vocal cordotomy. Moreover, surgical management of bilateral vocal fold paralysis is always a compromise between airway and voice. It is better to under correct the airway initially and perform a revision later if airway was found to be inadequate, as over correcting the airway initially will leave the patient with poor voice and reversal of over correction is difficult even under best circumstances.22 Conventional posterior cordotomy is done under general anesthesia it is difficult even for the very experienced surgeons to judge or limit the extent of cordotomy for achieving comfortable airway without leaving weak voice. In the present technique as the Awake laser posterior cordotomy is done under local anesthesia it has the advantage of monitoring voice during surgery and decide the extent of cordotomy needed to avoid over correction of glottic airway and weak voice. The present study for the first time describes the efficacy of Awake office based flexible endoscopic posterior cordotomy using diode laser under local anesthesia as an alternative to micro suspension cordotomy for bilateral vocal fold paralysis.

\section{Material and methods}

This retrospective study was done from January 2012 to January 2017 at Sri Sathya Sai E.N.T Hospital and research Center for Voice disorders, Guntur, A.P, India. The study was carried out on 20 patients of bilateral abductor vocal fold paralysis including $12(60 \%)$ females and $8(40 \%)$ males in the age group of 25 and 75 years. The present new technique of office based flexible endoscopic posterior cordotomy using diode laser under local anaesthesia was done for the first time at Sri Sathya Sai E.N.T Hospital and research Center for Voice disorders, Guntur, A.P, India, in2012as an alternative procedure to micro suspension cordotomy for a patient of BVFP with high risk for general anesthesia due to associated the cardiomy opathy and our early experience was presented in 2013. Subsequently patients of BVFP with other causes of comorbidity leading to either Difficult Laryngeal Exposure or High Risk for General Anaesthesia were operated by this new technique. Patients with other causes of vocal fold immobility and children were excluded from the study. Ethical approval for this study was granted by Institutional Ethical Committee of Sri Sathya Sai ENT Hospital and patient's acceptance obtained through informed consent. All the patients were given six to eight months time interval after the onset of bilateral vocal fold paralysis for the possibility of spontaneous recovery. All patients were evaluated with detailed case history, Video laryngostroboscopy (Figures1\&2) and flexible laryngoscopy and HRCT scan from skull base to chest. All the patients were followed at interval of one week for one month and later every 3 months for 9 months to 1 year after the surgery for respiration, phonation, and swallowing. The tracheotomized patients (7) were decannulated at an average of 2 to 4 weeks after the operation. Results were based on the Pulmonary Function Tests (PFT)and Voice Handicap Index( VHI) scores recorded in the pre and post operative period.Specific data included from pulmonary function tests was Peak Inspiratory Flow rate (PIF). PIF was measured through the mouth with the patient's nose and tracheostomy site (if any) occluded. The PIF value taken from the inspiratory phase of the flow-volume loop was considered as the critical indicator of glottal resistance to inspiratory flow. All patients showed improvement of dyspnea after the operation except 2 patients who required revision cordotomyas office procedure for more glottic widening and comfortable breathing which was performed 2 weeks after the primary procedure

\section{Surgical technique}

All the patients were operated in office based setup in lying and supine position for maintaining good haemodynamic stability, for avoiding vasovagal syncope, and for easy surgical maneuvers while the surgeon standing at the head end of the patient (Figure 3). Premedication given was, Glycopyrrolate $0.004 \mathrm{mg} / \mathrm{kg}$ I.M in all cases, and, Midazolam1 to 2mg I.M added in patients when associated with anxiety 30 minutes prior to the surgery. Both nostrils, oropharynx and glottis were sprayed with 4\% Lidocaine.Until 2015 we were using Fiber optic Flexible Laryngoscopes and later on chip on tip Video Flexible laryngoscopes with working channel. Endoscope was passed 
through the wider nostril, and $0.5 \%$ Lidocaine with 1 in $1,00,000$ epinephrine was injected in the vocal fold planned for cordotomy. Following all the Laser protective measures and using $980 \mathrm{~nm}$ Diode Laser, $300 \mathrm{mu}$ Laser fiber was passed through the working channel of the endoscope. Unilateral cordotomy was done with the contact Diode laser fiber applying 4 to 6 watts power by making a transverse incision of the vocal fold anterior to the vocal process and extended for about 4 to $5 \mathrm{~mm}$ laterally to the thyroid lamina cutting the elastic cone and vocalis muscle (Figure 4). Patient was asked to phonate intermittently during cordotomy and once the voice started becoming breathy, cordotomy was stopped and watched for respiratory status. When the patient had comfortable breathing (with Tracheotomy closed if present) further glottic widening was avoided to conserve the voice. Dexamethasone $8 \mathrm{mg}$ was given I.V intraoperatively. Oral antibiotics for 1 week and Anti -gastro esophageal reflux treatment for 3 months were given postoperatively. Patients were advised to leave the Office 2 hours after the procedure. Patients were followed every week for 1month, every month for 3 months and finally after 1 year. For patients with prior tracheotomy decannulation was done2 to 3 weeks postoperatively. Voice rest was advised for 1 week and voice therapy initiated 4 weeks following the surgery.

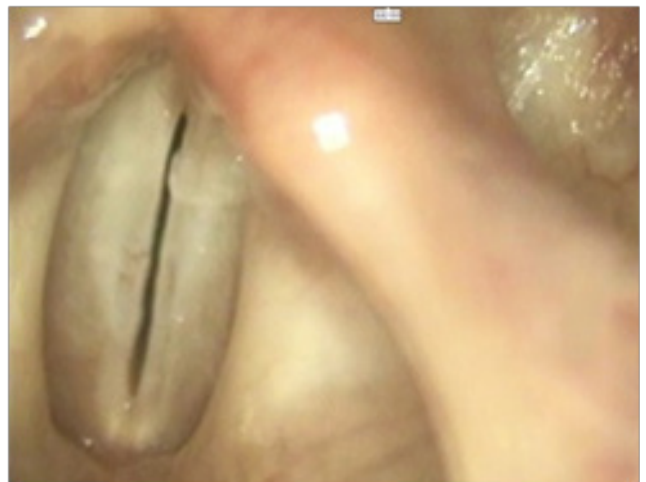

Figure I Preoperative $90^{\circ}$ laryngostroboscopy showing blateral vocal fold paralysis (BVFP).

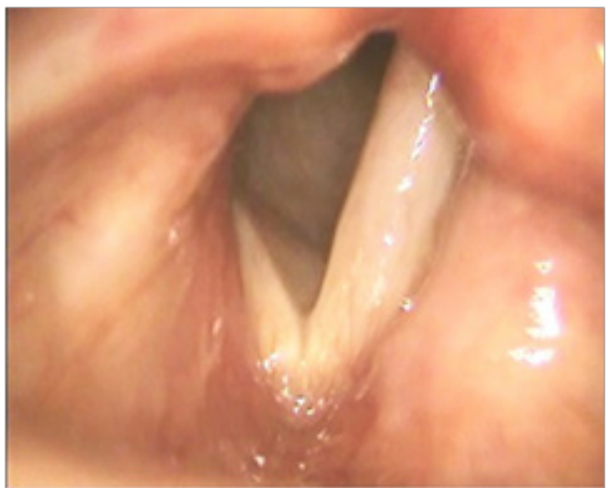

Figure 2 Postoperative $90^{\circ}$ laryngostroboscopy showing widening of the posterior glottic region I month after office based cordotomy.

\section{Results}

Assessment of respiration was based on the pre and postoperative pulmonary function test, Peak Inspiratory Flowrate (PIF) and assessment of voice was based on Voice Handicap Index (VHI) scores which were statistically significant as observed by paired T- test PIF ( $\mathrm{P}$ value $<.00001)$ and $(\mathrm{VHI})$ scores $(\mathrm{P}$ value $<.00003)$.

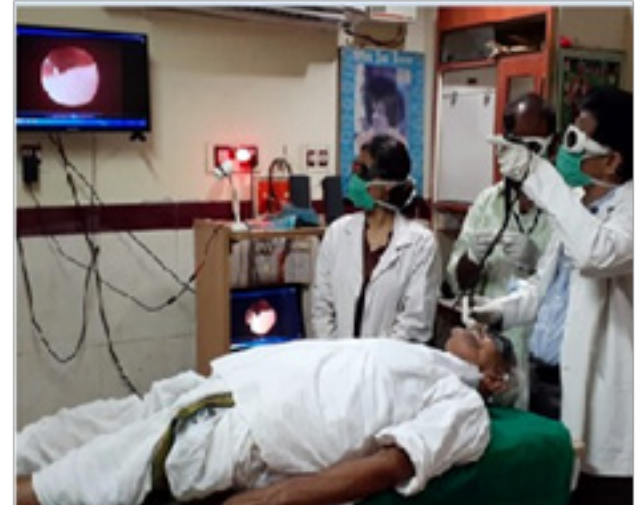

Figure 3 Showing patient lying in supine position and surgeon standing at the head end of the patient while performing office based cordotomy.

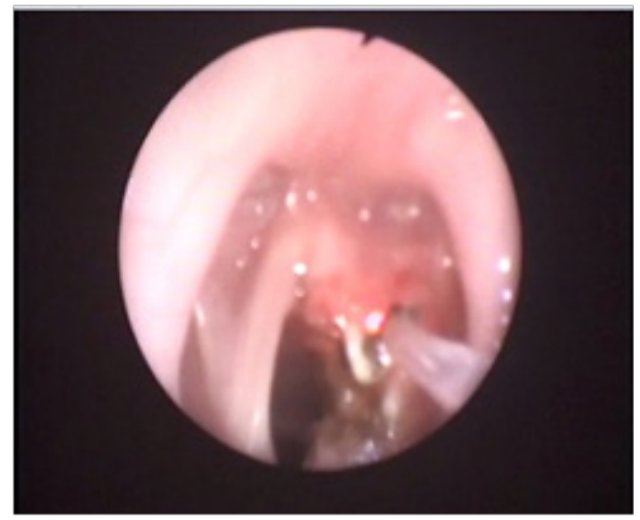

Figure 4 Intra-operative view showing office based cordotomy using diode laser for BVFP.

\section{Respiration}

Aerodynamic parameters showed statistically significant improvements between the pre- and post-operative period.

Specific data included from pulmonary function tests was Peak Inspiratory Flow rate (PIF) (Table 1). The PIF value taken from the inspiratory phase of the flow-volume loop was considered the critical indicator of glottal resistance to inspiratory flow. Postoperative respiratory function was adequate for the daily routine activities as walking and doing minor work, but not for the hard working activities as lifting heavy weights. There was no stridor or during sleep. All the patients showed improvement from dyspnea after the operation except 2 patients who required revision cordotomy as office procedure for more glottic widening and comfortable breathing which was performed 2 weeks after the primary procedure.

\section{Phonation}

To assess the voice outcome after the operation, we used the subjective assessment of patients for their voices by Voice Handicap Index ${ }^{19}$ (VHI after translation into Native Telugu language) at interval of 3 months, and 9 months after the operation (Table 2). There was gradual improvement in subjective voice parameters post-operatively, as assessed by the voice handicap index. After three months, 13 patients presented with mild dysphonia, 7 patients with moderate dysphonia . After 9 months, 16 cases with mild dysphonia and 4 
cases with moderate dysphonia. All the patients were satisfied with their voices after the operation. Only one patient, suffered from mild aspiration to fluids which resolved after three weeks and there were no other serious complications.

Table I Change in pulmonary inspiratory flow rates PIFR (1/sec) following cordotomy--P value $<.00001$

\begin{tabular}{llll}
\hline S. no & $\begin{array}{l}\text { PIFR-pre- } \\
\text { op }\end{array}$ & PIFR- post-op & PIFR increase \\
\hline 1 & 1.36 & 1.54 & 0.18 \\
2 & 0.69 & 0.95 & 0.26 \\
3 & 1.18 & 1.5 & 0.32 \\
4 & 1.12 & 1.71 & 0.59 \\
5 & 1.45 & 1.87 & 0.42 \\
6 & 0.71 & 1.22 & 0.51 \\
7 & 1.14 & 1.26 & $0.24 \mathrm{~s}$ \\
8 & 0.42 & 0.93 & 0.51 \\
9 & 0.44 & 0.96 & 0.52 \\
10 & 1.31 & 1.84 & 0.53 \\
11 & 1.57 & 2.03 & 0.46 \\
12 & 0.80 & 0.94 & 0.14 \\
13 & 0.93 & 1.95 & 1.02 \\
14 & 0.81 & 1.25 & 0.44 \\
15 & 1.01 & 1.24 & 0.23 \\
16 & 0.28 & 1.49 & 0.21 \\
17 & 0.62 & 1.21 & 0.59 \\
18 & 1.14 & 1.78 & 0.64 \\
19 & 0.56 & 1.17 & 0.61 \\
20 & 0.36 & 0.88 & 0.52 \\
\hline
\end{tabular}

Table $2 \mathrm{VHI}$ scores 3 months, and 9 months after cordotomy-- $\mathrm{P}$ value $<.00003$

\begin{tabular}{lll}
\hline Case no & After 3 months & $\begin{array}{l}\text { After } 9 \\
\text { months }\end{array}$ \\
\hline 1 & 34 & 28 \\
2 & 40 & 29 \\
3 & 33 & 28 \\
4 & 30 & 27 \\
5 & 32 & 26 \\
6 & 42 & 30 \\
7 & 39 & 26 \\
8 & 37 & 28 \\
9 & 34 & 27 \\
10 & 28 & 26 \\
11 & 43 & 30 \\
12 & 24 & 22 \\
13 & 32 & 27 \\
14 & 36 & 25 \\
15 & 25 & 23 \\
16 & 42 & 28 \\
17 & 34 & 22 \\
18 & 40 & 29 \\
19 & 36 & 24 \\
20 & 26 & 22 \\
\hline
\end{tabular}

\section{Discussion}

Numerous surgical procedures were proposed from the time of Chevalier Jacks on since 1922 for BVFP. Except emergency tracheotomy any procedure to treat this condition is better to be postponed for at least six months after the onset of paralysis for the chance of spontaneous recovery. Endoscopic Micro suspension $\mathrm{Co}_{2}$ laser posterior cordotomy under general anaesthesia has been proved as an effective and satisfactory procedure in treatment of bilateral vocal fold abductor paralysis. Next to $\mathrm{CO}_{2}, 980 \mathrm{~nm}$ diode laser is a new technology that has been successfully used in endolaryngeal microsurgery with better haemostatic properties. However limitations for endomicrolaryngeal surgical procedures were described in patients either with difficult laryngeal exposure or with high risk for general anaesthesia.The scope for the office-based laryngological procedures has expanded in the last decade since the advent of the distal-chip camera and new laser technology. In such situations the present innovative technique of Awake office based flexible endoscopic diode laser assisted Posterior Cordotomy under local anesthesia can be a suitable alternative to conventional micro suspension endoscopic vocal cordotomy. The present innovative technique of Awake office based flexible endoscopic diode laser assisted posterior cordotomy under local anesthesia has the advantages of monitoring and conserving voice during the procedure and performing revision cordotomy as a office procedure. As regards respiratory outcome 18 patients $(92.3 \%)$ in this study improved after the operation. Only 2 patients $(7.7 \%)$ did not improve and needed revision cordotomy as office procedure. However, the percentage of second intervention reached up to $33 \%$ in the study done by Dennis and Kashima ${ }^{8}$ Regarding the voice outcome, we used the Voice Handicap Index (VHI) scores which were also followed in the previous studies. ${ }^{22}$ In this study, all of our patients were satisfied with their voices. 17 patients $(84.6 \%)$ presented with moderate dysphonia three months after the operation. After 9 months, 20 patients $(100 \%)$ presented with mild dysphonia. Similar to any other office laryngeal procedure the technique has limitations in patients with severe gag reflex, severe anxiety, movement disorders and in children.

\section{Conclusion}

Unilateral Micro suspension $\mathrm{CO} 2$ laser posterior cordotomy is a simple and a fast technique that has been proved as a satisfactory procedure in treatment of bilateral vocal fold abductor paralysis because of its short operative time, satisfactory results, and low complication incidence. Micro suspension $\mathrm{CO}_{2}$ cordotomy under general anesthesia is difficult to apply in patients with difficult neck or high risk for general anesthesia due to associated comorbidity. In such occasions Awake office based transnasal flexible endoscopic diode laser assisted posterior cordotomy can be considered as a perfect alternative to micro suspension $\mathrm{CO}_{2}$ cordotomy. These procedures are well tolerated by patients, cost effective with minimal morbidity. Recovery time is minimized and the risk of minor complications is less than $1 \%$. Conservative and planned cordotomy is possible by Awake laser cordotomy done under local anesthesia by monitoring the voice during procedure for a compromise between comfortable airway and acceptable voice. Revision cordotomy by Awake laser cordotomy as an office based procedure under local anesthesia, is safe and cost effective and much easier compared to revision $\mathrm{CO} 2$ cordotomy procedure under general anesthesia in the operating room. In the present study the new technique of Awake Office based Flexible Endoscopic Posterior cordotomy using Diode Laser is confined to the patients of BVFP associated with comorbid conditions leading to difficulty for Micro suspension cordotomy. To proove it's efficacy in 
the day to day surgical management of bilateral vocal fold paralysis needs further studies. A larger series over a longer follow up period will further help to accurately assess the efficacy of this procedure.

\section{Acknowledgments}

None.

\section{Conflict of interest}

The author declares that there is no conflict of interest to disclose.

\section{Funding}

None.

\section{References}

1. Benninger MS, Gillen JB, Altman JS. Changing etiology of vocalfimmobility. Laryngoscope. 1998;108(9):1346-1350.

2. Raghavendra Rao, Vijendra Shenoy, Vishnu Prasad, et al. A comparative study of laser posterior cordotomy and vocal cord lateralization. Egyptian Journal of Ear, Nose, Throat and Allied Sciences. 2015;16(3):255-258.

3. Jackson C. Ventriculocordectomy. A new operation for the cure of goitrous glottic stenosis. Arch Surg. 1922;4(2):257-274.

4. King BT. A new and function restoring operation for bilateral abductor cord paralysis. JAMA. 1939;112(9):814-823.

5. Ejnell. A simple operation for bilateral vocal cord paralysis. Laryngoscope. 1984;94(7):954-95.

6. Thornell WC. Intralaryngeal approach for arytenoidectomy in bilateral abductor vocal cord paralysis. Arch Otolaryngol. 1948;47(4):505-508.

7. Ossoff RH, Karlan MS, Sisson GA. Endoscopic laser arytenoidectomy. LasersSurg Med. 1983;2(4):293-299.

8. Dennis DP, Kashima H. Carbon dioxide laser posterior cordectomy for treatment of bilateral vocal cord paralysis. Ann Otol Rhinol Laryngol. 1989;98:930-934.

9. Joel A Ernster, Arlen D Meyers. Bilateral Vocal Fold Paralysis. $2009 ; 25(3)$.

10. Cagatay Oysu, Sema Zer Toros, Cxig־dem Tepe-Karaca, et al. Endoscopic Posterior Cordotomy with Microdissection Radiofrequency Electrodes for Bilateral Vocal Cord Paralysis. Otolaryngology- Head and Neck Surgery. 2014;150(1):103106.
11. Kyra M Osborne, Paul C Bryson, Michael S Benninger. Cordotomy by Coblation in Bilateral Vocal Fold Immobility. Otolarngology-Head and Neck Surgery. 2012;147(2).

12. Mahmoud A Khalil, Hazem M Abdel Tawab. Laser Posterior Cordotomy: Is it a Good Choice in Treating Bilateral Vocal Fold Abductor Paralysis? Clin Med Insights Ear Nose Throat. 2014;7:13-17.

13. Fanjul M, García Casillas MA, ParenteA, etal.Utilizacióndelláser diodo en la vía aérea pediátrica. Cir Pediatr. 2008;21(2):79-83.

14. Yan Y, Olszewski AE, Hoffman MR, et.al. Use of lasers in laryngeal surgery. $J$ Voice. 2010; 24(1):102-109.

15. Saetti R, SilvestriniM, GaliottoM et al. Contactlaser surgery in treatment of vocal fold paralysis. Acta Otorhino laryngol Ital. 2003;23(1):33-37.

16. Emanuele Ferri, García Purriños FJ. Diode laser surgery in the endoscopic treatment of laryngeal paralysis. Acta Otorrinolaringológica Española. 2006;57(6):270-4.

17. Oswal VH, Gandhi SS. Endoscopic laser management of bilateral abductor palsy. Indian Otolaryngol Head Neck Surg. 2009;61 (Suppl 1):47-51.

18. Viwanath B, Arlen D Mayers. Vocal Cord Cordotomy. Drugs and Diseases Clinical procedures, Medscape. 2015.

19. Joshua B, Feinmesser R, Zohar L, et.al Endoscopic laser assisted posterior ventriculocordectomy without tracheostomy for bilateral vocal cord immobility. Isr Med Assoc J. 2004;6(6):336338.

20. Veluswamy Anand, Kumaran BR, Chenniappan S. Cordoplasty: A New Technique for Managing Bilateral Vocal Cord Paralysis and Its Comparison with Posterior Cordotomy and External Procedure in a Large Study Group. Indian J Otolaryngol Head Neck Surg. 2015; 67(Suppl 1):40-46.

21. Benninger MS, Bhattacharyya N, Fried MP. Surgical management of bilateral vocal fold paralysis. Oper Tech Otolaryngol. 1998;9(4):224-229.

22. Barbara H. Jacobson, Alex Johnson, et al. The Voice Handicap Index (VHI): Development and Validation. American Journal of Speech-Language Pathology. 1997;6(3):66-70. 